\title{
Silicon-vacancy color centers in phosphorus-doped diamond
}

\author{
Assegid Mengistu Flatae ${ }^{\mathrm{a}, *}$, Stefano Lagomarsino ${ }^{\mathrm{a}, \mathrm{b}}$, Florian Sledz ${ }^{\mathrm{a}}$, Navid Soltani ${ }^{\mathrm{a}}$, Shannon S. Nicley ${ }^{\mathrm{c}, \mathrm{d}}$, \\ Ken Haenen ${ }^{c}$, Robert Rechenberg ${ }^{\mathrm{e}}$, Michael F. Becker ${ }^{\mathrm{e}}$, Silvio Sciortino b,f, Nicla Gelli ${ }^{\mathrm{b}}$, Lorenzo Giuntini ${ }^{\mathrm{b}, \mathrm{f}}$, \\ Francesco Taccetti $^{\mathrm{b}}$, Mario Agio ${ }^{\mathrm{a}, \mathrm{g}}$
}

a Laboratory of Nano-Optics and C $\mu$, University of Siegen, 57072 Siegen, Germany

${ }^{\mathrm{b}}$ Istituto Nazionale di Fisica Nucleare, Sezione di Firenze, 50019 Sesto Fiorentino, Italy

${ }^{c}$ Institute for Materials Research (IMO) \& IMOMEC, Hasselt University \& IMEC vzw, 3590 Diepenbeek, Belgium

${ }^{\mathrm{d}}$ Department of Materials, University of Oxford, Parks Road, Oxford OX1 2PH, United Kingdom

e Fraunhofer USA Center for Coatings and Diamond Technologies, East Lansing, MI 48824, USA

${ }^{\mathrm{f}}$ Dipartimento di Fisica e Astronomia, University of Florence, 50019 Sesto Fiorentino, Italy

g National Institute of Optics (INO), National Research Council (CNR), 50125 Florence, Italy

\section{A B S T R A C T}

The controlled creation of color centers in phosphorus-doped (n-type) diamond can facilitate the electronics integration of quantum photonics devices, such as single-photon sources operating upon electrical injection. Silicon vacancy (SiV) color centers are promising candidates, but so far the conditions for single-photon emission in phosphorus-doped diamond have not been investigated. In this study, we create SiV color centers in diamond samples with different phosphorus concentrations and show that the fluorescence background due to doping, nitrogen-impurities and ion implantation induced defects can be significantly suppressed. Single-photon emitters in phosphorus-doped diamond are obtained at the low Si-ion implantation fluences.

\section{Introduction}

Efficient and scalable solid-state single-photon sources operating at room temperature are crucial to the development of quantum photonics technologies, such as quantum computers, secure communication lines and precision measurements below the shot-noise limit [1-3]. In these respects, optically and electrically driven semiconductor quantum dots exhibit the best performances, but they require operation at cryogenic temperatures [4-7]. Under ambient conditions, color centers in diamond may represent an alternative platform. In particular, silicon vacancy $(\mathrm{SiV})$ color centers have most of the fluorescence emission $(>90 \%)$ concentrated in a narrow zero-phonon line at room temperature [8]. In addition, the center exhibits a short excited-state lifetime and a small inhomogeneous linewidth broadening [8].

It has been recently shown that the electrical excitation of $\mathrm{SiV}$ color centers is determined by electron and hole-capture processes, which are directly related to the density of free electrons and holes in the vicinity of the color center [9]. This possibility has been recently demonstrated using p-i-n based diode structures [10,11], where the Si-ions are implanted in the intrinsic layer. However, the creation of $\mathrm{SiV}$ color centers in p- or n-type diamond can avoid the need for the complex semiconductor junctions. For example, SiV color centers in n-type dia- mond allow the implementation of a Schottky-diode. This would give the possibility of injecting holes directly from the metal contact and of utilizing electrons from the n-type diamond host lattice. In recent years, n-type conduction in diamond has been successfully obtained in phosphorous (P)-doped diamond grown by microwave plasma chemical vapor deposition (CVD) [12-15].

However, there are challenges to attain the conditions for single-photon emission in P-doped diamond. The crystal imperfections (structural distortion and degradation caused by the incorporation of phosphorus atoms during the growth process [16-21]), phosphorus-based bound excitons [22] and phosphorus-related complex defects [23,24], photoluminescence related to phosphorus-nitrogen co-doping $[23,25]$ and ion-beam induced defects can compromise the signal-to-noise ratio required for single-photon emission.

Previous photoluminescence experiments on P-doped diamond revealed broad background emission assigned to donor-acceptor-pair recombination although the nature of the defects was not clear. CVD diamond crystal usually contains a high concentration of vacancies, as high as $10^{18} \mathrm{~cm}^{-3}$ [26], and some form of phosphorus and vacancy related bound up is a possibility. The lattice damage caused during ion implantation can be partially healed by annealing. Its effectiveness in favoring Frenkel couples recombination that avoid the formation of va-

\footnotetext{
* Corresponding author.

E-mail address: flatae@physik.uni-siegen.de (A.M. Flatae)
} 
cancy complexes have been reported for p-type (boron-doped) diamond [27], but reproducible results in P-doped diamond have not yet been reported [28]. Therefore, attaining single-photon emission from SiV color centers in P-doped diamond is not obvious.

Here, we investigate the optical properties of $\mathrm{SiV}$ color centers in P-doped single-crystal diamond under different implantation and doping parameters, namely Si-ions implanted at a fluence range of $10^{7}-10^{14} \mathrm{~cm}^{-2}$ and phosphorus concentrations in the gas feed of $4300 \mathrm{ppm}, 5000 \mathrm{ppm}$ and 20,000 ppm. We show that the luminescence background can be significantly suppressed even for high phosphorus doses and that the conditions for single-photon emission can be attained.

\section{Samples}

Three P-doped single-crystal diamond films were grown on different high-pressure-high-temperature (HPHT) diamond substrates by microwave plasma-enhanced CVD (MWPECVD) using phosphine $\left(\mathrm{PH}_{3}\right)$ as a dopant source.

One of the P-doped singe crystalline diamond thin film was grown on a (111) oriented HPHT diamond substrate with lateral dimensions of $2.6 \mathrm{~mm} \times 2.6 \mathrm{~mm}$ (sample A). Prior to deposition, the growth surface was mechanically polished using a high speed scaife. The P-doped diamond was grown utilizing an in-house built $2.45 \mathrm{GHz}$ MWPECVD reactor at a pressure of 160 Torr and $2 \mathrm{~kW}$ absorbed microwave power. The resulting deposition temperature was $940{ }^{\circ} \mathrm{C}$, as measured by a single color IR-pyrometer $(\varepsilon=0.6)$. The hydrogen $\left(\mathrm{H}_{2}\right)$ rich plasma contained $0.09 \%$ of methane $\left(\mathrm{CH}_{4}\right)$ with a $\mathrm{PH}_{3} / \mathrm{CH}_{4}$ ratio of $4300 \mathrm{ppm} .4 \mathrm{~h}$ of deposition resulted in a smooth diamond film with a thickness of $2.0 \mu \mathrm{m} \pm 0.1 \mu \mathrm{m}$ measured by linear encoder (Solartron DR600).

The other two P-doped homo epitaxial samples were grown similarly on (111) oriented HPHT diamond substrates with lateral dimensions of $2.5 \mathrm{~mm} \times 2.5 \mathrm{~mm}$ (Sumitomo). Both samples were grown by MWPECVD in a $2.45 \mathrm{GHz}$ ASTeX PDS17 reactor with a water cooled molybdenum substrate holder. A $500 \mathrm{sccm}$ total flow rate and pressure of 140 Torr were held constant. The $\mathrm{H}_{2}$ plasma contained $0.15 \% \mathrm{CH}_{4}$. One of the samples (sample B) was grown with a P-gradient, with the $\mathrm{PH}_{3} / \mathrm{CH}_{4}$ ratio adjusted in eight one hour-steps, of $0,250,500,1000$, $2500,5000,10,000$, and 20,000 ppm, respectively. The other sample (sample C) was grown with a constant $5000 \mathrm{ppm} \mathrm{PH}_{3} / \mathrm{CH}_{4}$. The applied forward microwave power was varied between 1.0 and $1.3 \mathrm{~kW}$ to maintain a substrate growth temperature of $1000{ }^{\circ} \mathrm{C}$. The substrate temperature was also monitored by tungsten vanishing filament pyrometry assuming an emission coefficient of $\epsilon=0.6$. Both samples were grown for $8 \mathrm{~h}$ of total deposition time. The $\mathrm{H}_{2}$ and $\mathrm{CH}_{4}$ gasses are filtered to $<1 \mathrm{ppb}(9 \mathrm{~N})$ purity, and the $\mathrm{PH}_{3}$ is used from a source diluted to $200 \mathrm{ppm}$ in $\mathrm{H}_{2}$. The sample morphologies of the films were investigated by optical microscopy and the thicknesses were measured by linear encoder (Mitutoyo 542-158), with nine measurements at the sample center, with the thickness error estimated as the average of the standard errors before and after growth. Sample B was grown to a thickness of $1.3 \mu \mathrm{m} \pm 0.2 \mu \mathrm{m}$ and sample $\mathrm{C}$ to $2.2 \pm 0.2 \mu \mathrm{m}$.

The three P-doped samples were used as a host matrix for the creation of SiV color centers. The Si-ions implantation is based on a $3 \mathrm{MeV}$ Tandetron accelerator (High Voltage Engineering Europe) equipped with a HVEE 860 Negative Sputter Ion Source. This allows for the acceleration of ion species $\left(\mathrm{Si}^{+}, \mathrm{Si}^{2+}, \mathrm{Si}^{3+}\right)$ in the range of few $\mathrm{MeV}$ energies depending on the terminal voltage and the ion charge state. Aluminum (Al) metal foils were used to decrease the ion energy down to a few tens of keV for shallow implantation (within $200 \mathrm{~nm}$ from the surface). The possibility of using pulsed ion-beams allows control over the number of implanted ions in the desired place. A custom designed furnace $\left(1200{ }^{\circ} \mathrm{C}\right.$ in high-vacuum conditions $\left.\sim 10^{-7} \mathrm{mbar}\right)$ enables the activation of the SiV color centers in P-doped samples. The samples have been Si-implanted with five different fluences $\left(\sim 10^{14} \mathrm{~cm}^{-2}, \sim 10^{13} \mathrm{~cm}^{-2}\right.$, $\sim 10^{12} \mathrm{~cm}^{-2}, \sim 10^{8} \mathrm{~cm}^{-2}$ and $\sim 10^{7} \mathrm{~cm}^{-2}$. The ex- pected implantation depth is $\leq 200 \mathrm{~nm}$. Details on the implantation process can be found in Ref. [29]. To optically characterize the samples, spectrally and temporally resolved $\mu$-photoluminescence measurements are conducted in a homemade confocal microscopy setup equipped with a Hanbury-Brown Twiss interferometer for the verification of single-photon emission [8].

\section{Experimental results}

\subsection{Spectroscopy and discussion}

Sample A was implanted with four different Si-ion fluences of $10^{8} \mathrm{~cm}^{-2}, 10^{12} \mathrm{~cm}^{-2}, 10^{13} \mathrm{~cm}^{-2}$ and $10^{14} \mathrm{~cm}^{-2}$. Unlike for intrinsic single-crystal diamond [8], fluence-dependent background at the emission wavelength of the $\mathrm{SiV}$ color centers is evident, especially for the higher implantation fluences, as depicted in Fig. 1a. The peak at $708 \mathrm{~nm}$ corresponds to the Raman line of diamond excited by a $647-\mathrm{nm}$ laser. Using two band pass filters, the SiV color centers' emission spectral window is selected and the decay kinetics are measured. The fluorescence lifetime shows a background-related slow component (8.0-8.6 ns) and a fast component corresponding to the excited-state lifetime of the $\mathrm{SiV}$ (0.6-1.3 ns). Fig. 1b shows the measurement in different parts of the sample. The implanted area has a non-uniform ion distribution (hence the damage is not uniform) because of the nearly Gaussian profile of the ion-beam and the ion scattering on the diaphragms limiting its size $[8,29]$, which leads to a position-dependent background and SiV luminescence. For instance, the black curve in Fig. 1b shows the contribution of $\mathrm{SiV}$ color centers (fast component) and the background (slow component) in the $10^{14} \mathrm{~cm}^{-2}$ ion-implanted region. In another region of the sample, the background emission is so high that it completely dominates the SiV signal and the lifetime exhibits mainly the slow component. Fig. 1b (red curve) shows the fluorescence lifetime measurement of a background-dominated region implanted with $10^{13} \mathrm{~cm}^{-2}$. The region implanted with $10^{8} \mathrm{~cm}^{-2}$ shows relatively low background, comparable to the region where no ions are implanted. Fig. 1c shows the emission from the color centers (black curve) and the background (bg) far away from the implantation spot (blue curve).

Unlike for higher implantation fluences, the fluorescence decay kinetics in the region implanted with $10^{8} \mathrm{~cm}^{-2}$ exhibits a single exponential decay with a time constant of $1.0 \mathrm{~ns}$, corresponding to the excited-state lifetime of the SiV in intrinsic single-crystal diamond [8]. In the region with the lowest implantation fluence, the emitters are well separated as shown in the wide field image of Fig. 1d inset. The emitters, however, do not exhibit anti-bunching behavior, indicating a possible clustering of two or more centers. With such implantation fluence, the expected ions density is $\sim 1 \mu \mathrm{m}^{-2}$. Taking into consideration a few percent activation yield (1-4\% [8]), clustering of SiV centers is evident. Color center clustering is common at the grain boundaries of polycrystalline diamond, but in a P-doped diamond crystal the unavoidable presence of defects (dislocations) can be a possibility [30]. We have also observed the analogous phenomenon for $\mathrm{SiV}$ color centers in intrinsic single-crystalline diamond [8,29].

The ion-beam fluence dependent background is further investigated by different laser excitations ( $532 \mathrm{~nm}, 647 \mathrm{~nm}, 656 \mathrm{~nm}$ and $690 \mathrm{~nm}$ ) and we attribute its main source to the creation of nitrogen-vacancy (NV) related impurities. A higher fluence of implanted Si-ions creates more vacancies and favors the formation of NV color centers during annealing. Fig. 2a shows the photoluminescence spectra in the region implanted with a fluence of $10^{12} \mathrm{~cm}^{-2}, 10^{13} \mathrm{~cm}^{-2}$ and $10^{14} \mathrm{~cm}^{-2}$ using $532 \mathrm{~nm}$ CW laser excitation at $2 \mathrm{~mW}$. The increase in the emission of $\mathrm{NV}$ color centers with increasing Si-ion implantation fluence is clearly observed. Spatially- and spectrally-resolved confocal scanning images using a high numerical microscope objective (Zeiss, C EpiPlan-Apochromat, $50 \times / 0.95$ DIC) show that the NV color centers are mainly created at the location of SiV color centers, as depicted in Fig. 2b. A 532 

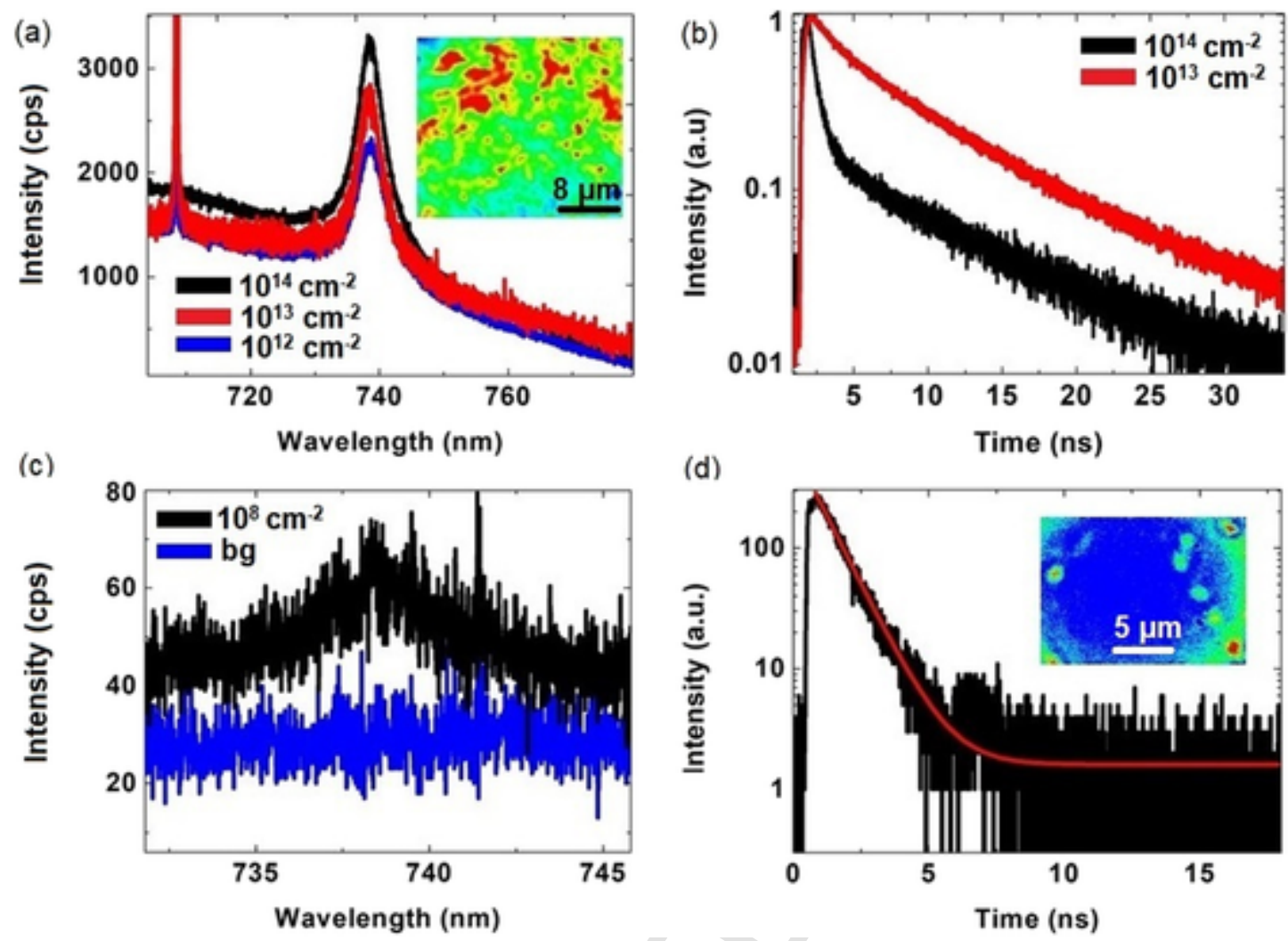

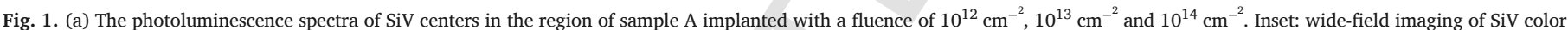

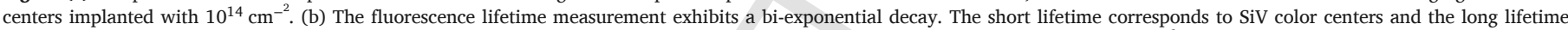

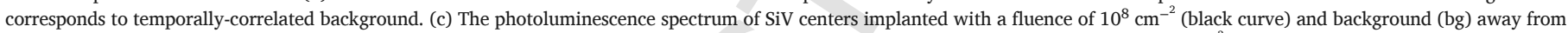

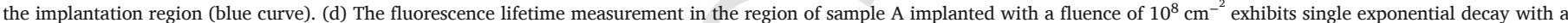

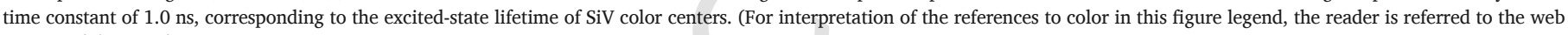
version of this article.)

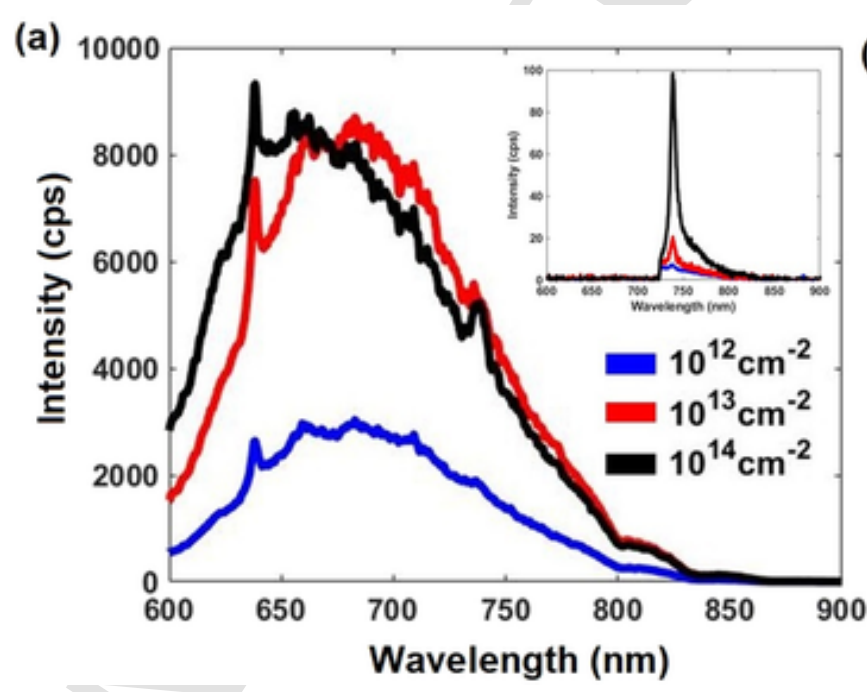

(b)

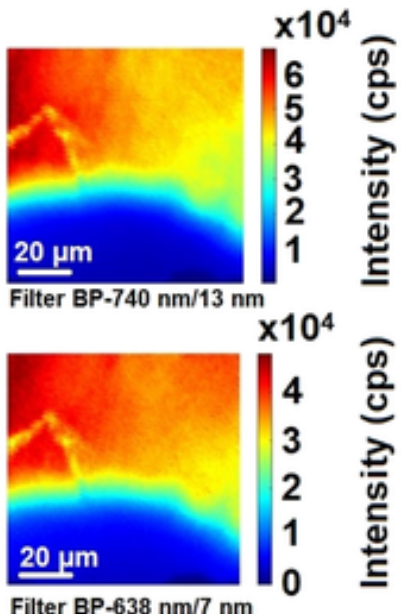

Filter $\mathrm{BP}-638 \mathrm{~nm} / 7 \mathrm{~nm}$

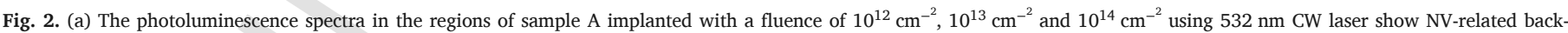

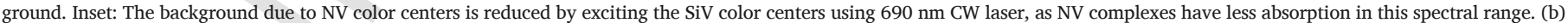

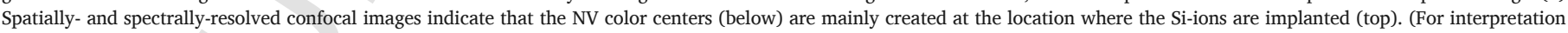
of the references to color in this figure, the reader is referred to the web version of this article.)

nm CW laser excites the sample and the emission from the sample is sent to two avalanche photodiodes (APDs) (Micro Photonic Devices). The signal that goes to one APD is filtered for the detection of SiV emission using a bandpass filter (BP-740 nm/13 nm), while the other APD detects the emission from $\mathrm{NV}$ color centers using another bandpass filter (BP-638 nm/7 nm). Since both signals are collected at the same time for a given point (with a lateral resolution of $300 \mathrm{~nm}$ ), the one-to-one correspondence between the $\mathrm{SiV}$ and NV signals confirms our claim. As shown in Fig. 2b, the region implanted with Si-ions exhibits the emission of both $\mathrm{SiV}$ and NV-color centers (red color). The blue color depicts the region where the ions are not implanted. The background due to $\mathrm{NV}$ color centers is reduced by exciting the $\mathrm{SiV}$ color centers us- 
ing $656 \mathrm{~nm}$ and $690 \mathrm{~nm}$ CW laser, as NV complexes have less absorption in this spectral range. Fig. 2a inset shows the photoluminescence spectra of the color centers using $690 \mathrm{~nm}$ CW laser excitation (at $1 \mathrm{~mW}$ ). Spectral measurements and confocal images show that by using the $690 \mathrm{~nm}$ excitation wavelength the background signal associated with $\mathrm{NV}$ color centers is reduced by two orders of magnitude, while the SiV color centers signal-to-noise ratio is enhanced by an order of magnitude as compared to the excitation at $532 \mathrm{~nm}$.

The fluorescence lifetime of NV- color centers in bulk diamond is $\sim 12 \mathrm{~ns}$ as stated in the literature, but there are works reporting values around $8 \mathrm{~ns}$ [31], where samples with a high nitrogen concentration are considered. In our case, the change in the lifetime is attributed to a different host medium (as it is doped with phosphorous), to higher non-radiative relaxation channels resulting from defect related quenching, and to a higher concentration of nitrogen [31].

Similar implantation dependent background is also observed for sample B. This sample was implanted with Si-ion fluences of $\sim 10^{12} \mathrm{~cm}^{-2}$ and $\sim 10^{7} \mathrm{~cm}^{-2}$. Even if the background is not as pronounced as in the previous sample (sample A), the photoluminescence spectrum and the fluorescence lifetime measurements confirm the trend. Fig. 3a depicts the photoluminescence spectra of the color centers in different parts of the sample. Due to the Gaussian-like profile of the implanted ion distribution, the color centers in the region at the tail of the $10^{7} \mathrm{~cm}^{-2}$ Si-ion beam implantation exhibit less background (Fig. 3a blue curve and inset). The background level and the signal-to-

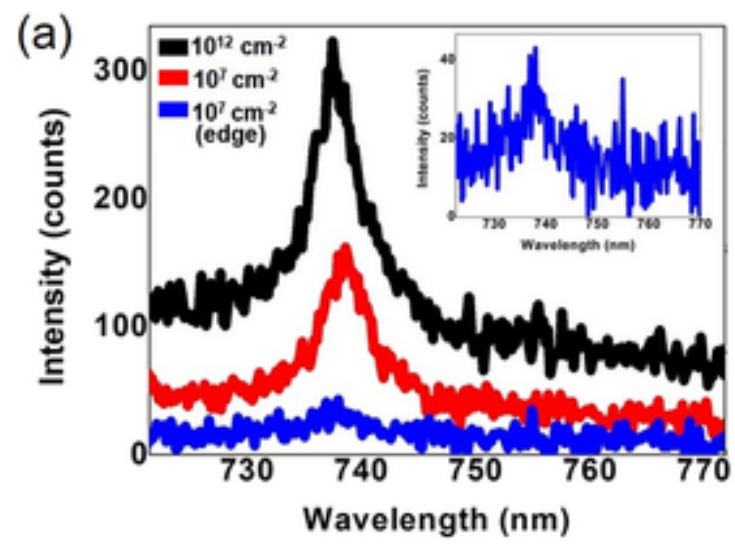

noise ratio of the $\mathrm{SiV}$ color centers are similar to those in intrinsic single-crystal diamond excited under similar conditions [8]. In the highly implanted region (Fig. 3a, black curve) the background is still observable and its temporal contribution is present in the fluorescence decay curve as shown in Fig. 3b. The slow component is attributed to the background and the fast one to the excited-state lifetime of the SiV (0.8 ns).

For further investigation of $\mathrm{SiV}$ color centers in P-doped diamond and for attaining single-photon emission, sample $\mathrm{C}$ was implanted with Si-ions at four different fluences $\left(\sim 10^{14} \mathrm{~cm}^{-2}, \sim 10^{13} \mathrm{~cm}^{-2}, \sim 10^{12} \mathrm{~cm}^{-2}\right.$ and $\sim 10^{7} \mathrm{~cm}^{-2}$ ). Spectral measurements show that the implanted regions exhibit less background (Fig. 4a) and, where the implanted ions are fewer $\left(10^{7} \mathrm{~cm}^{-2}\right.$ and in the tail of a region implanted with $10^{12} \mathrm{~cm}^{-2}$ ), the signal is dominated by SiV emission. The inset in Fig. $4 \mathrm{a}$ shows the emission of the SiV color centers (blue curve) and the background (bg) away from the implanted region (black curve). The background in this region is not temporally-correlated and leads a mono-exponential fluorescence decay kinetics, which corresponds to the excited-state lifetime of $\mathrm{SiV}$ color centers (1.13 ns), as shown in Fig. 4b. This sample is further investigated for single-photon emission as discussed in the next session.

The decrease in background is mainly attributed to the creation of less NV color centers. Further investigation of the sample using $532 \mathrm{~nm}, 647 \mathrm{~nm}, 656 \mathrm{~nm}$ and $690 \mathrm{~nm}$ laser confirms our claim. Fig. 5 a shows the photoluminescence spectra in the regions of sample $C$ implanted with a fluence of $10^{12} \mathrm{~cm}^{-2}, 10^{13} \mathrm{~cm}^{-2}$ and $10^{14} \mathrm{~cm}^{-2}$ us-

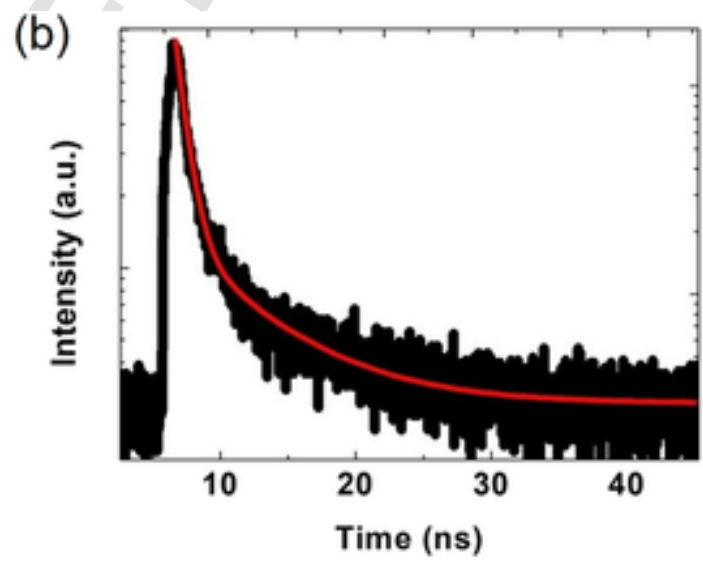

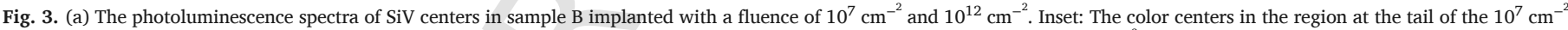

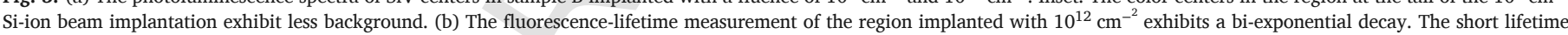

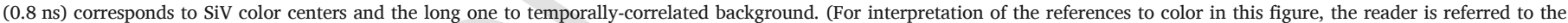
web version of this article.)

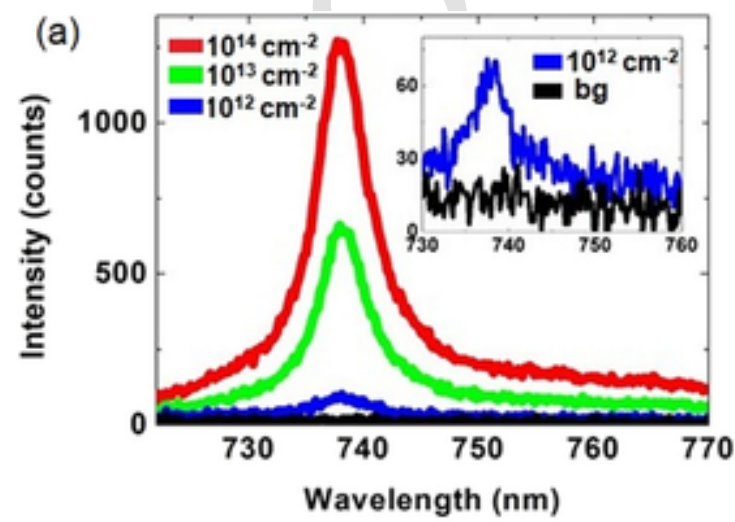

(b)

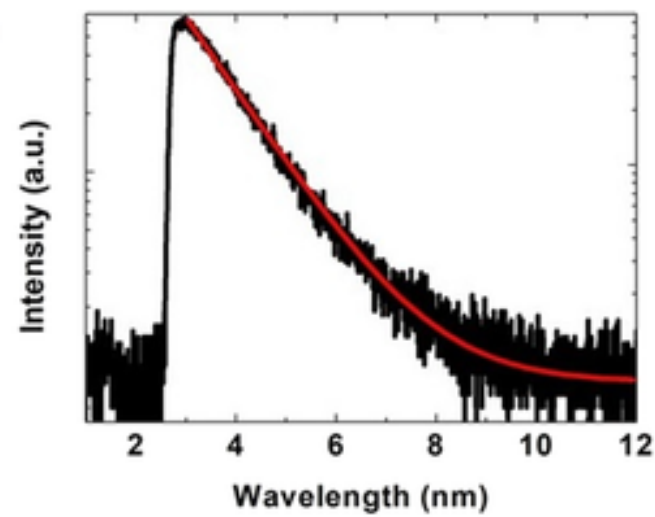

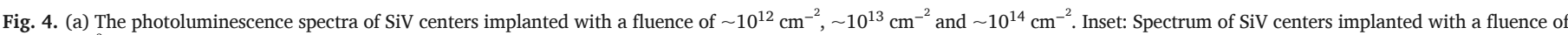

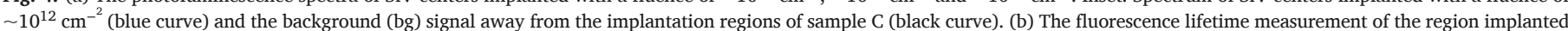

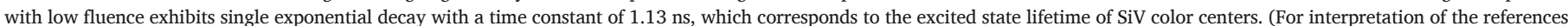
to color in this figure legend, the reader is referred to the web version of this article.) 
(a)

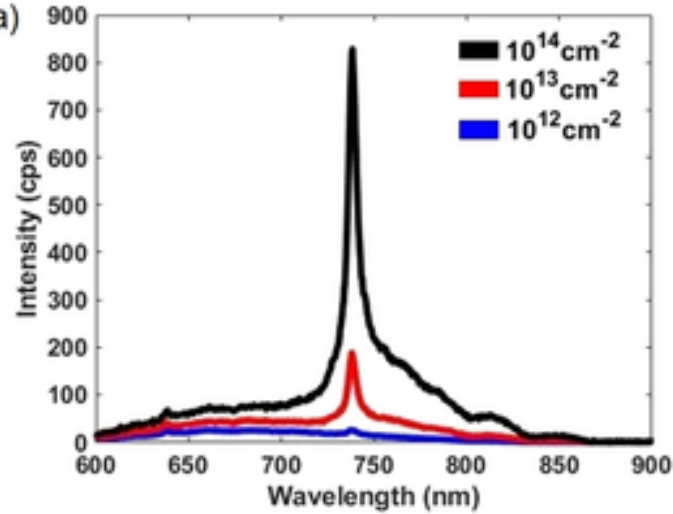

(b)

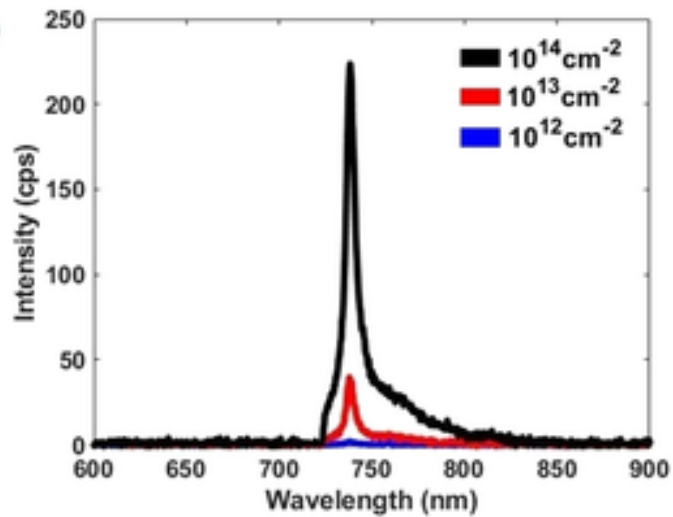

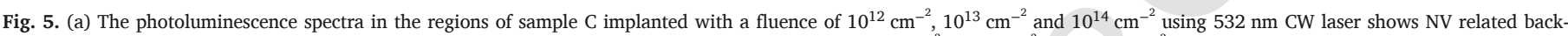

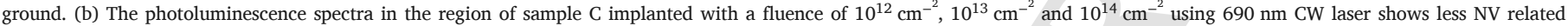
background.

ing $532 \mathrm{~nm}$ CW laser excitation at $2 \mathrm{~mW}$. The increase in the contribution of NV color centers with increasing in Si-ion implantation fluence is clearly observed, but less pronounced as compared to sample A. The background due to NV color centers was further reduced by exciting the $\mathrm{SiV}$ color centers using $656 \mathrm{~nm}$ and $690 \mathrm{~nm}$ CW laser. Fig. 5b shows the photoluminescence spectra of the color centers using $690 \mathrm{~nm}$ CW laser excitation at $1 \mathrm{~mW}$.

In general, as shown clearly in the temporally- and spectrally-resolved measurements, sample $\mathrm{C}$ has relatively less background (supposedly due to less nitrogen content) and it is further investigated for single-photon emission as discussed in the next session. Moreover, temperature dependent electrical measurements on this sample (not shown) demonstrate the conductivity of the P-doped film. The sample ex- hibits a resistance of nearly $300 \mathrm{M} \Omega$ at room temperature, which drops to $75 \mathrm{M} \Omega$ when the sample is heated to around $160{ }^{\circ} \mathrm{C}$. These measurements are in agreement with a theoretical model presented in Ref. [32].

\subsection{Single-photon emission}

A wide-field imaging in the tail of a region implanted with $10^{12} \mathrm{~cm}^{-2}$ and in the region implanted with the lower fluence $10^{7} \mathrm{~cm}^{-2}$ of sample C shows well separated SiV color centers, as depicted in Fig. 6a. Each of them is investigated and the signal-to-noise ratio of a $\mathrm{SiV}$ center is optimized for the detection of single photons. The fluorescence signal of the measured $\mathrm{SiV}$ color centers is concentrated in

(b)

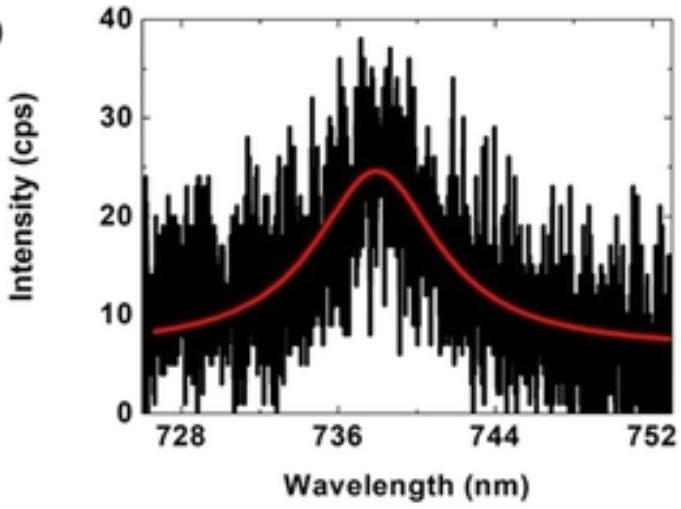

(d)
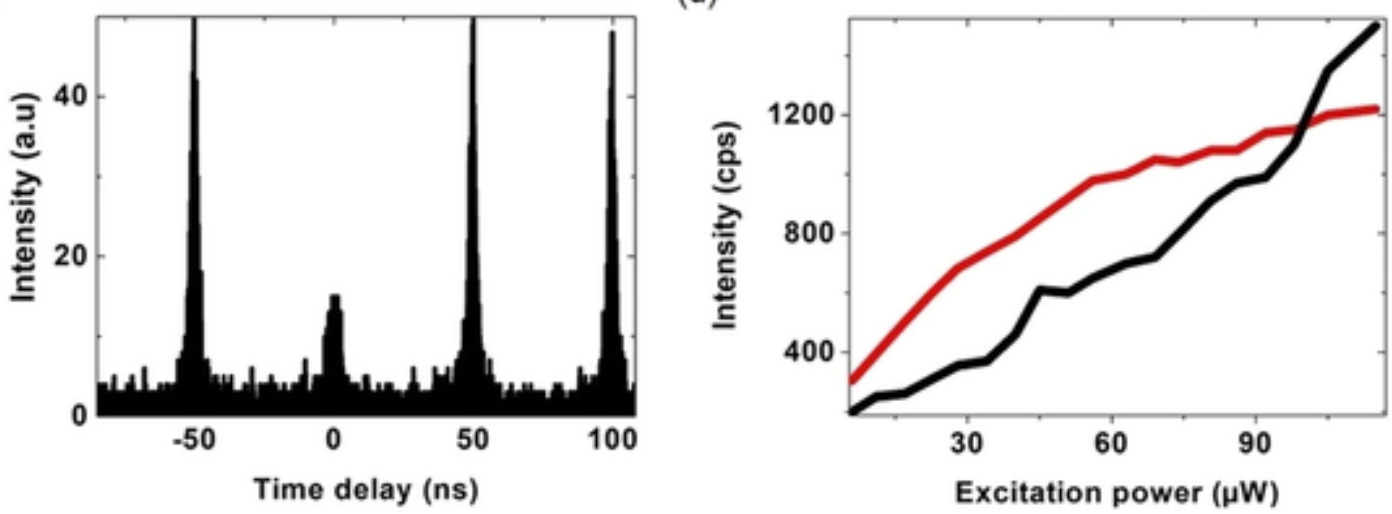

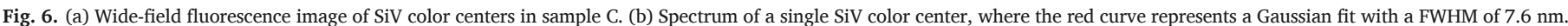

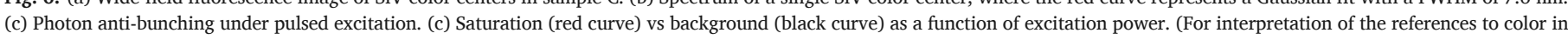
this figure legend, the reader is referred to the web version of this article.) 
the narrow zero-phone line at $737.96 \pm 0.05 \mathrm{~nm}$, with a room temperature linewidth down to $6.0 \pm 1.8 \mathrm{~nm}$. Ten SiV color centers are used to acquire statistical information. Fig. $6 \mathrm{~b}$ shows the spectrum of a single SiV color center. A Gaussian fit (red curve) yields a full-width at half-maximum (FWHM) of $7.6 \mathrm{~nm}$ centered at $737.95 \mathrm{~nm}$. This SiV color center has been identified as a single-photon source by measuring the 2nd order intensity autocorrelation function $g^{2}(\tau)$ of the emitted photons in pulsed excitation (pulse width $<90 \mathrm{ps}$ ), as a function of the time delay $\tau$. At the repetition rate of $20 \mathrm{MHz}, \mathrm{g}^{2}(\tau)$ exhibits individual fluorescence pulses spaced by the pulse period with a missing one at $\tau$ equal to zero, which confirms a single-photon source (Fig. 6c). Since due to electronic and photonic noises, $\mathrm{g}^{(2)}(0)$ cannot be exactly zero, a commonly accepted signature of a single-photon source is $g^{(2)}(0)<0.5$. Fig. 6c reveals the observation of a single-photon source in a P-doped diamond sample with $g^{2}(0)=0.3$. To the best of our knowledge, this is the first observation of single-photon emission from this kind of system. Most of the emitters shown in Fig. 6a do not show an anti-bunching behavior. This is attributed to the tendency of the activated centers to cluster. This is also manifested by the observation of higher count rates as compared to single $\mathrm{SiV}$ color centers. The presence of defects (e.g. dislocations) around which the formation of a SiV center could be energetically favored could be an explanation. These points are currently under investigation. By counting the number of SiV color centers and determining the occurrence of clustering, the activation yield for the creation of $\mathrm{SiV}$ color centers amounts to $3.5 \pm 0.5 \%$. This number is similar to our previous report on the activation yield of $\mathrm{SiV}$ in intrinsic single-crystalline CVD diamond [8].

The intensity-dependent background of the sample prevents the observation of single-photon emission at high excitation power (above saturation). The red curve in Fig. 6d shows the count rate of the SiV color center as a function of the excitation power. The count rates are background corrected (including the detector's dark counts). The fluorescence count rate I of an emitter as a function of the excitation power $P$ is described as $I=I_{\infty} /\left(1+P_{\text {sat }} / P\right)+C_{b s} P$, where $P_{\text {sat }}$ is the excitation power at saturation and $I_{\infty}$ is the maximum photon count rate. The background $C_{b g} P$ is measured in the nearby region, where there is no $\mathrm{SiV}$ color center (black curve). Using this equation, we found a maximum photon count rate of $I_{\infty}=1.576 \pm 0.035 \times 10^{3} \mathrm{cps}$ (at $P_{\text {sat }}=36 \mu \mathrm{W} \pm 3 \mu \mathrm{W}$ ), which corresponds to about $\sim 4 \times 10^{5}$ cps taking into account light trapping in diamond and the overall detection efficiency of our setup. This number corresponds to the count rate of SiV color centers in intrinsic bulk diamond [8]. The count rate (hence the signal-to-noise ratio) of the color centers can be improved by selecting emitters with an appropriate dipole moment orientation. The possibility of growing thin P-doped diamond films would allow the implementation of Yagi-Uda antennas for highly efficient light extraction and directional emission [33].

\section{Conclusions}

In conclusion, we have reported the creation and optical characterization of SiV color centers in P-doped diamond samples and have shown that the fluorescence background due to doping, nitrogen-impurities and ion-implantation induced defects can be significantly suppressed for attaining single-photon emission with high spectral quality. The most critical parameter is the nitrogen concentration in P-doped diamond, which should be below $1 \mathrm{ppb}$ in the technical gases during CVD growth. The possibility of accessing individual SiV color centers in P-doped diamond paves the way to the implementation of simpler configurations for the electrical excitation of diamond-based single-photon sources, e.g. Schottky diodes in place of p-i-n junctions, hence facilitating the integration of quantum photonic devices in diamond-based electronics.

\section{CRediT authorship contribution statement}

Assegid Mengistu Flatae: Investigation. Stefano Lagomarsino: Investigation. Florian Sledz: Investigation. Navid Soltani: Investigation. Shannon S. Nicley: Investigation. Ken Haenen: Investigation. Robert Rechenberg: Resources. Michael F. Becker: Resources. Silvio Sciortino: Investigation. Nicla Gelli: Investigation. Lorenzo Giuntini: Investigation. Francesco Taccetti: Investigation. Mario Agio: Conceptualization, Methodology, Supervision.

\section{Declaration of competing interest}

The authors declare that they have no known competing financial interests or personal relationships that could have appeared to influence the work reported in this paper.

\section{Acknowledgements}

The authors gratefully acknowledge financial support from the University of Siegen, the German Research Association (DFG) (INST 221/118-1 FUGG, 410405168), the Hasselt University Special Research Fund (BOF), the Research Foundation Flanders (FWO), and the Methusalem NANO network. SSN is a Newton International Fellow of the Royal Society. The authors also acknowledge INFN-CHNet, the network of laboratories of the INFN for cultural heritage, for support and precious contributions in terms of instrumentation and personnel. A.M. Flatae and M. Agio would like to thank F. Tantussi, F. De Angelis for experimental support and D. Yu. Fedyanin for helpful discussions. This work is based upon networking from the COST Action MP 1403 "Nanoscale Quantum Optics," supported by COST (European Cooperation in Science and Technology).

\section{References}

[1] T D Ladd, F Jelezko, R Laflamme, Y Nakamura, C Monroe, J L O’Brien, Quantum computers, Nature 464 (2010) 45.

[2] V Giovannetti, S Lloyd, L Maccone, Advances in quantum metrology, Nat. Photonics 5 (2011) 222.

[3] A V Sergienko, Quantum Communications and Cryptography, CRC, Boca Raton, FL, 2005.

[4] Z Yuan, B E Kardynal, R M Stevenson, A J Shields, C J Lobo, K Cooper, N S Beattie, D A Ritchie, M Pepper, Electrically Driven Single-photon Source Science, 295, 2002, p. 102.

[5] D J P Ellis, A J Bennett, S J Dewhurst, C A Nicoll, D A Ritchie, A J Shields, Cavity-enhanced radiative emission rate in a single photon-emitting diode operating at $0.5 \mathrm{GHz}$, New J. Phys. 10 (2008) 043035.

[6] M J Conterio, N Sköld, D J P Ellis, I Farrer, D A Ritchie, A J Shields, A quantum dot single photon source driven by resonant electrical injection, Appl. Phys. Lett. 103 (2013) 162108.

[7] T Heindel, C Schneider, M Lermer, S H Kwon, T Braun, S Reitzenstein, S Höfling, M Kamp, A Forchel, Electrically driven quantum dot-micropillar single photon source with 34\% overall efficiency, Appl. Phys. Lett. 96 (2010) 011107.

[8] S Lagomarsino, A M Flatae, S Sciortino, F Gorelli, M Santoro, F Tantussi, F De Angelis, N Gelli, F Taccetti, L Giuntini, M Agio, Optical properties of silicon-vacancy color centers in diamond created by ion implantation and post-annealing, Diam. Relat. Mater. 84 (2018) 196.

[9] D Yu Fedyanin, M Agio, Ultrabright single-photon source on diamond with electrical pumping at room and high temperatures, New J. Phys. 18 (2016) 073012.

[10] A M Berhane, S Choi, H Kato, T Makino, N Mizuochi, S Yamasaki, I Aharonovich, Electrical excitation of silicon-vacancy centers in single crystal diamond, Appl. Phys. Lett. 106 (2015) 171102.

[11] B Tegetmeyer, C Schreyvogel, N Lang, W Müller-Sebert, D Brink, C E Nebel, Elec troluminescence from silicon-vacancy centers in diamond p-i-n diodes, Diam. Relat. Mater. 65 (2016) 42-46.

[12] S Koizumi, M Kamo, Y Sato, H Ozaki, T Inuzuka, Growth and characterization of phosphorus doped $\{111\}$ homoepitaxial diamond thin films, Appl. Phys. Lett. 71 (1997) 1065.

[13] S Koizumi, T Teraji, H Kanda, Phosphorus-doped chemical vapour deposition of diamond, Diam. Relat. Mater. 9 (2000) 935.

[14] T A Grotjohn, D T Tran, M K Yaran, S N Demlow, T Schuelke, Heavy phosphorus doping by epitaxial growth on the (111) diamond surface, Diamond Relat. Mater. 44 (2014) 129.

[15] Y Balasubramaniam, P Pobedinskas, S D Janssens, G Sakr, F Jomard, S Turner, Y-G Lu, W Dexters, A Soltani, J Verbeeck, J Borjon, M Nesladek, K Haenen, Thick homoepitaxial (110)-oriented phosphorus doped n-type diamond, Appl. Phys. Lett. 109 (2016) 62105

[16] S Bohr, R Haubner, B Lux, Influence of phosphorus addition on diamond CVD, Diam. Relat. Mater. 4 (113) (1995). 
[17] N Orita, T Nishimatsu, H K Yoshida, Ab initio study for site symmetry of phosphorus-doped diamond, Japan, J. Appl. Phys. 46 (2007) 315.

[18] J P Goss, P R Briddon, R Jones, S Sque, Donor and acceptor states in diamond, Diam. Relat. Mater. 13 (2004) 684.

[19] S J Sque, R Jones, J P Goss, P R Briddon, Shallow donors in diamond: Chalcogens, Pnictogens, and their hydrogen complexes, Phys. Rev. Lett. 92 (2004) 017402.

[20] J Isoya, M Katagiri, T Umeda, S Koizumi, H Kanda, N T Son, A Henry, A Gali, E Janzen, Pulsed EPR studies of phosphorus shallow donors in diamond and SiC, Physica 376 (2006) 358.

[21] H Kato, T Makino, S Yamasaki, H Okushi, n-Type diamond growth by phosphorus doping on (001)-oriented surface, J. Phys. D. Appl. Phys. 40 (2007) 6189.

[22] J Barjon, Luminescence spectroscopy of bound excitons in diamond, Phys. Status Solidi A 214 (2017) 1700402.

[23] J te Nijenhuis, S M Olsthoorn, W J P van Enckevort, L J Giling, Red luminescence in phosphorous-doped chemically vapor deposited diamond, J. Appl. Phys. 82 (1997) 419.

[24] R Jones, J E Lowther, J Goss, Limitations to n-type doping in diamond: the phosphorus-vacancy complex, Appl. Phys. Lett. 69 (1996) 2489.

[25] G Z Cao, F A J M Driessen, G J Bauhuis, L J Giling, P F A Alkemade, Homoepitaxial diamond films codoped with phosphorus and nitrogen by chemical-vapor deposition, J. Appl. Phys. 78 (1995) 3125.

[26] S Dannefaer, W Zhu, T Bretagnon, D Kerr, Vacancies in polycrystalline diamond films, Phys. Rev. B 53 (1996) 1979.
[27] J F Prins, Activation of boron-dopant atoms in ion-implanted diamonds, Phys. Rev. B 38 (1988) 5576-5584.

[28] R Kalish, Ion implantation in diamond for quantum information processing (QIP): doping and damaging, Quantum Information Processing With Diamond, Principles and Applications, Woodhead Publishing, 2014, pp. 36-67.

[29] S Lagomarsino, S Sciortino, N Gelli, A M Flatae, F Gorelli, M Santoro, M Chiari, C Czelusniac, M Massi, F Taccetti, M Agio, L Giuntini, The center for production of single-photon emitters at the electrostatic deflector line of the Tandem accelerator of LABEC (Florence), Nucl. Inst. Methods Phys. Res. B 422 (2018) 31.

[30] A Tallaire, T Ouisse, A Lantreibecq, R Cours, M Legros, H Bensalah, J Barjon, V Mille, O Brinza, J Achard, Identification of dislocations in synthetic chemically vapour deposited diamond single crystals, Cryst. Growth Des. 16 (2016) 2741

[31] E Fraczek, V G Savitski, M Dale, B G Breeze, P Diggle, M Markham, A Bennett, H Dhillon, M E Newton, A J Kemp, Laser spectroscopy of NV- and NV0 colour centres in synthetic diamond, Opt. Mater. Express 7 (2017) 2571.

[32] I A Khramtsov, D Yu Fedyanin, Superinjection in diamond p-i-n diodes: bright single-photon electroluminescence of color centers beyond the doping limit, Phys. Rev. Appl. 12 (24013) (2019).

[33] H Galal, A M Flatae, S Lagomarsino, G Schulte, C Wild, E Wörner, N Gelli, S Sciortino, H Schönherr, L Giuntini, M Agio, Highly efficient light extraction and directional emission from diamond color centers using planar Yagi-Uda antennas, arXiv:1905.03363 (2019). 